\title{
A uniqueness theorem for surfaces in the large
}

\author{
By Samuel I. GoldBERG
}

(Received February 18, 1976)

\section{Introduction}

It has long been conjectured that among the compact (connected) oriented surfaces in $E^{3}$ only the sphere has constant mean curvature $\mu$. This was established by $\mathrm{H}$. Hopf [4] for surfaces of genus zero. For arbitrary genus a proof was given by A. D. Aleksandrov [1] under the assumption that there are no self intersections, i.e., for surfaces embedded in $E^{3}$. Recently S. -S. Chern and the author [2] showed that a closed orientable surface of constant $\mu$ such that $K^{2} \geqq C / 2 \mu^{2}$ is a sphere. ( $K$ is the Gaussian curvature and $C$ is a nonnegative scalar invariant of the Gauss map of the immersed surface.) In this note the following theorem is obtained by a similar method.

ThEOREM. A closed orientable surface in $E^{3}$ of constant mean curvature $\mu$ with $K\left(\mu^{2}-K\right) \geqq-C / 4 \mu^{2}$ is a sphere.

T. Klotz and R. Osserman [6] proved that a complete surface immersed in $E^{3}$ with constant $\mu$ and $K \geqq 0$ is a sphere, a plane, or a right circular cylinder. Our method also yields a new proof of this since under the conditions $K$ is a superharmonic function. (Observe that in the Theorem, $K$ is not assumed to be nonnegative.)

\section{Harmonic Mappings}

To prove the theorem, the well-known fact that the Gauss map of a surface $S$ in $E^{3}$ is harmonic if the mean curvature is constant, is used. $A$ brief review of the theory as given in [3] is now presented.

Let $M$ and $N$ be smooth oriented Riemannian manifolds of dimensions $m$ and $n$, respectively, with the metrics $d s_{M}^{2}$ and $d s_{N}^{2}$, and let $f: M \rightarrow N$ be a smooth mapping. Locally, then, $d s_{M}^{2}=\sum \omega_{i}^{2}$ and $d s_{N}^{2}=\sum \omega_{a}^{*^{2}}$, where the $\omega_{i}, i=1, \cdots, m$ and $\omega_{a}^{*}, a=1, \cdots, n$ are linear differential forms in $M$ and $N$, respectively. (Corresponding quantities in $N$ are denoted with an asterisk.) We write $f^{*} \omega_{a}^{*}=\sum A_{i}^{a} \omega_{i}$. The covariant differential $D$ of $A_{i}^{a}$ is defined by

$$
D A_{i}^{a} \equiv d A_{i}^{a}+\sum_{j} A_{j}^{a} \omega_{j i}+\sum_{b} A_{i}^{b} \omega_{b a}^{*}=\sum_{j} A_{i j}^{a} \omega_{j} \quad \text { (say) }
$$


with $A_{i j}^{a}=A_{j i}^{a}$, where the $\omega_{j i}$ and $\omega_{b a}^{*}$ are the connection forms of $d s_{M}^{2}$ and $d s_{N}^{2}$, respectively. (In the sequel, the symbol $f^{*}$ is omitted from such formulas with no resulting confusion.) The mapping $f$ is said to be harmonic if $\sum A_{i i}^{a}=0$.

Taking the exterior derivative of (2.1) and using the structure equations in $M$ and $N$, we get if $f$ is harmonic, the Laplacian

$$
\sum_{k} A_{i k k}^{a}=\sum_{k} A_{k}^{a} R_{k i}-\sum_{b, c, a, k} R_{b a c l}^{*} A_{k}^{b} A_{k}^{c} A_{i}^{d},
$$

where $R_{k i}$ is the Ricci tensor of $d s_{M}^{2}$ and $R_{b a c d}^{*}$ is the curvature tensor of $d s_{N}^{2}$. Let $u=\sum\left(A_{i}^{a}\right)^{2}$. Then, its Laplacian $\Delta u=\sum u_{k k}$ is given by

$$
\frac{1}{2} \Delta u=\sum_{a, i, j}\left(A_{i j}^{a}\right)^{2}+\sum_{a, i, j} R_{i j} A_{i}^{a} A_{j}^{a}-\sum_{\substack{a, b, c, d \\ i, j}} R_{a b c d}^{*} A_{i}^{a} A_{j}^{b} A_{i}^{c} A_{j}^{a} .
$$

The details of the proof of this formula may be found in [3].

The last term in (2.2) may be expressed as

$$
\sum R_{a b c d}^{*} A_{i}^{a} A_{j}^{b} A_{i}^{c} A_{j}^{d}=2 \sum_{i<j} R^{*}\left(A_{i}, A_{j}\right)\left\|A_{i} \wedge A_{j}\right\|^{2},
$$

where $A_{i}$ is the local vector field with components $\left(A_{i}^{1}, \cdots, A_{i}^{n}\right)$ and $R^{*}\left(A_{i}, A_{j}\right)$ denotes the sectional curvature of $N$ along the section spanned by $A_{i}$ and $A_{j}$ at each point.

\section{Proof of the Theorem}

Let $M=S, N=$ the unit sphere in $E^{3}$ with the constant curvature metric, and let $f: S \rightarrow N$ be the Gauss map. In this case, $A_{i}^{a}=h_{i a}, i, a=$ 1,2 , where the $h_{i a}$ are the coefficients of the second fundamental form of the immersion. Since $\mu=$ const., $f$ is a harmonic map. Noting that $2 \mu=$ $h_{11}+h_{22}, K=h_{11} h_{22}-\left(h_{12}\right)^{2}, u=4 \mu^{2}-2 K$ and $A_{i j}^{a}=h_{i j a}$, the formula (2.2) yields after an elementary computation,

$$
-\Delta K=4 K\left(\mu^{2}-K\right)+\frac{C}{\mu^{2}}
$$

where $C$ is the nonnegative scalar invariant of $f$ given by $C=\mu^{2} \sum\left(h_{i j k}\right)^{2}$. For, the r.h.s. of $(2.3)$ equals $2 K^{2}$ since $R^{*}\left(A_{i}, A_{j}\right)=1$ and $\sum_{i<j}\left\|A_{i} \wedge A_{j}\right\|^{2}$ $=K^{2}$. By hypothesis, $K\left(\mu^{2}-K\right) \geqq-C / 4 \mu^{2}$, so $S$ being compact, $K$ must be a positive constant. Thus, $S$ is a sphere.

It was shown in [2] that on a surface of constant mean curvature in $E^{3}$ the function $\log K^{2}$ is superharmonic wherever $K \neq 0$. However, we were unable to obtain a geometrical interpretation of this fact.

If $K \geqq 0$ and $\mu=$ const., formula (3.1) says $K$ is a superharmonic function. We assume $S$ is complete. If $K \equiv 0$, formula (3.1) says that 
$S$ is either a plane or a right circular cylinder. If $K \not \equiv 0$ and $S$ is compact, it is a sphere by the theorem. If $K \not \equiv 0$ and $S$ is not compact, 'Theorem 15 in A. Huber [5] says that $S$ is parabolic. It follows that $K$ is a constant greater than zero, so $S$ is compact, and we have a contradiction.

\section{References}

[1] A. D. AleKsandrov: Uuiqueness theorem for surfaces in the large $V$, Vestnik Leningrad Univ. Ser. Mat. Meh. Astronom. 13 (1958), 5-8.

[2] S. -S. CHERN and S. I. GOLDBERG: On the volume decreasing property of a class of real harmonic mappings, Amer. Journ. Math. 97 (1975), 133-147.

[3] S. I. GOLDBERG and T. IsHIHARA: Harmonic quasiconformal mappings of Riemannian manifolds, Amer. Journ. Math., 98 (1976), 225-240.

[4] H. HopF: Lectures on differential geometry in the large, mimeographed notes, Stanford University, 1956.

[5] A. HUBER: On subharmonic functions and differential geometry in the large, Comment. Math. Helv. 32 (1957), 13-72.

[6] T. Klotz and R. Osserman: Complete surfaces in $E^{3}$ with constant mean curvature, Comment. Math. Helv. 41 (1966-67), 313-318.

University of Illinois Urbana, Illinois 61801 